\title{
REPRESIÓN DE LAS RADIOS PIRATAS COMERCIALES EN EUROPA
}

\section{THE REPRESSION OF COMMERCIAL PIRATE RADIO STATIONS IN EUROPE}

Francisco José Montes Fernández: Profesor Facultad de Ciencias de la Información de la Universidad Complutense de Madrid. (España)

fimontes@telefonica.net

Javier Sierra Sánchez: Profesor Facultad de Comunicación de la Universidad San Jorge de Zaragoza. (España)

jsierra@usj.es

\section{CURRÍCULUM VITAE}

Licenciado en Ciencias de la Información. Es Profesor Asociado de Relaciones Internacionales en los medios audiovisuales en la Universidad Complutense de Madrid. En 1977 entra en la dirección de Relaciones Internacionales de RTVE, donde en 1981 es nombrado jefe del Departamento de Documentación.

Profesor de Teoría de la Comunicación de la Universidad San Jorge de Zaragoza (España). Doctor en Ciencias de la Información por la Universidad Complutense de Madrid (España). Licenciado en Comunicación Audiovisual y Periodismo por la Universidad Complutense de Madrid. Experto en Relaciones Públicas Internacionales por la Universidad Complutense de Madrid (España). Experto en 
Protocolo y ceremonial del estado e Internacional por la Universidad de Oviedo (España) y la Escuela Diplomática de Madrid (España).

Ha impartido numerosas ponencias en congresos y seminarios nacionales e internacionales. Ha participado en varios proyectos de investigación financiados por el Ministerio de Educación y Ciencia, MEC y por la Universidad Complutense de Madrid (España). Ha sido becario FPI de la Universidad Complutense de Madrid. Es miembro fundador de la revista científica Icono 14

\section{RESUMEN}

En este artículo nos aproximamos al estudio de las radios piratas comerciales. Por primera vez en la historia de la comunicación social se estudia este fenómeno desde la perspectiva universitaria. Acotamos en un primer momento el objeto de estudio y los tipos; para posteriormente abordar la importancia del fenómeno comunicativo, empresarial y político que tuvieron este tipo de emisoras. A través de la publicidad los dueños de estas estaciones radiofónicas articularon un modelo de negocio muy rentable y con una gran capacidad de persuasión sobre la población más joven. La importancia de las estaciones piratas comerciales en el contexto europeo fue tan grande que sus emisiones hicieron cambiar toda la programación de la radiodifusión de nuestro continente, pasando de difundir unos espacios serios y rigurosos, con música clásica, dramáticos, conferencias, etc. a una programación basada en la música ligera presentada por un nuevo tipo de locutor ("disk jockey"). En este artículo descubrimos de forma cronológica las fases y el porqué de la extinción de las estaciones piratas comerciales en Europa. 


\section{PALABRAS CLAVE}

Historia de la radiodifusión en Europa - Radiodifusión pirata comercial - Radio Caroline - Radio Véronica - Radio Mi Amigo - Radio Mercur

\section{ABSTRACT}

This article studies commercial pirate radio stations. For the first time in the history of social communication, we approach this phenomenon from the perspective of higher education. Firstly, we will delimit the object of study and its types then discuss the communicative, commercial and political relevance that this type of broadcasting had. By means of advertising, the owners of these radio stations developed an extremely profitable and persuasive business model, aimed at younger audiences. The influence of commercial pirate radio stations in Europe was so great that their broadcasting brought about radical changes in all radio broadcasting in Europe. Programmes based on pop music, presented by a new kind of presenter, the DJ, replaced serious, rigorous programmes broadcasting classical music, drama, debates etc.. In this article we take a chronological look at the phases in, and the reasons for, the extinction of commercial pirate radio stations in Europe.

\section{KEY WORDS}

History of radio broadcasting in Europe - Commercial pirate broadcasting - Radio Caroline - Radio Véronica - Radio Mi Amigo - Radio Mercur 


\section{ÍNDICE}

\section{Definición}

2. Acuerdo europeo para la represión de emisiones extraterritoriales.

3. Conclusiones

4. Bibliografía

4.1. Tratados, revistas, periódicos y acuerdos internacionales

\subsection{Libros}

5. Notas

\section{TEXTO}

\section{Definición}

Aguilera (1985: 66) atribuye el origen de la denominación al "carácter marítimo e ilegal de la primera emisora inglesa, al Acta de emisiones marítimas, promulgada por el gobierno laborista británico, que excluía a estas radios de la legalidad, y también al hecho de enarbolar la bandera pirata en los mástiles de los barcos que albergaron a algunas de ellas"

Jurídicamente, esto es con el Reglamento de Radiocomunicaciones a la vista, son estaciones ilícitas y públicamente apátridas -es en este sentido por el que cabe denominarlas "piratas"- las siguientes:

a) Las instaladas a bordo de barcos, aeronaves, islas artificiales y otros objetos flotantes o firmes que se encuentren fuera de los territorios nacionales (Reglamento 
Radiocomunicaciones Art. 7 n. 422, el art. 28 n. 962, añade: "A las estaciones móviles en el mar o por encima del mar, les está prohibido efectuar servicio alguno de radiodifusión".)

b) Las que efectúen transmisiones sin señal de identificación o utilicen una señal falsa (Reglamento Radiocomunicaciones Art. 19, nº.735, Art. 14 n. 693.) A estos efectos, el Reglamento de Radiocomunicaciones precisa que el indicativo debe ser de tal naturaleza "que pueda permitir la identificación internacional sin confusión posible". Es uso tradicional de la radiodifusión que tal indicativo haga referencia a la matrícula de la emisora (por ejemplo, "EAJ-1" -ver nota 1- ) y a su nombre ("Radio Barcelona"), con estos datos queda explícita o implícitamente notificada su denominación, nacionalidad, ubicación, la identidad del concesionario, señal o sintonía característica, etc. (Reglamento de Radiocomunicaciones Art. 19 nº 737)

c) Las que funcionen sin la correspondiente licencia (Reglamento de Radiocomunicaciones Art. $18 \mathrm{n}^{\mathrm{O}}$ 125) o las que operando con ella se presentan al público confusamente haciendo creer que están instaladas en un territorio para el que, sin duda, no están autorizadas a funcionar.

Las estaciones piratas comerciales pueden encasillarse en los tres órdenes (están instaladas en buques o islas artificiales situados fuera de territorios nacionales, su identificación no es completa porque induce o permite confusión y operan sin licencia), las políticas son básicamente del tipo b) y aunque cabe presumir que disponen de licencia en el territorio desde el que emiten, resultan internacionalmente ilícitas por falta de su frecuencia a la IFRB de la UIT. En efecto, el Reglamento de Radiocomunicaciones determina que "deberá notificarse a la Junta Internacional de Registro de Frecuencias toda asignación de frecuencia relativa a una estación... de radiodifusión,... b) si la frecuencia se utiliza para la radiocomunicación internacional" (Reglamento de Radiocomunicaciones Art. 9 nº.486.). Es evidente que la difusión 
internacional de programas desde un país a la población de otro constituye una radiocomunicación internacional.

Las estaciones piratas pueden ser: comerciales, políticas y libres. En este artículo nos vamos a centrar en el estudio de las medidas que se adoptaron para la represión de las radios piratas comerciales. Éstas se caracterizan por:

- están instaladas en buques o islas artificiales situados fuera de los territorios nacionales.

- su identificación no es completa porque induce o permite confusión y

- operan sin licencia.

- el objetivo prioritario de estas estaciones es el lucro.

\section{Acuerdo europeo para la represión de emisiones extraterritoriales}

Desde 1961 la UER tomó partida luchando contra las estaciones piratas en conjunción con el Consejo de Europa y la UIT, Para ello hizo un seguimiento en sus Comisiones Jurídicas, el tercer nivel de la Unión, los Consejos de Administración, el segundo órgano en importancia de la organización y en sus Asambleas Generales, el máximo organismo de la UER y donde se encuentran representados todos los miembros activos (los países europeos y los africanos ribereños del mediterráneo) y los miembros asociados (el resto de las organizaciones que pertenecían en aquel momento a la UER).

Entre las conclusiones de la Comisión se plantearon tres preguntas importantes: 
$1^{a}$ ¿La legislación de los países puede castigar actos en los que el resultado se toma fuera de las aguas territoriales?

En este sentido, se recurrió a la nueva Convención, el Reglamento de Radiocomunicaciones y sus anexos redactado en Ginebra en 1959, en el que se emplaza a los Estados a que tomen las medidas que impidan la explotación de estaciones de radiodifusión a bordo de buques o aeronaves. Los Estados firmantes de la Convención, el Reglamento y sus anexos representan el 90\% deben impedir la asistencia a las estaciones piratas. La misma obligación se impone por los principios generales del derecho de gentes, y especialmente por el derecho de buena vecindad y del principio más específico en las telecomunicaciones, según el cual los Estados deben poner fin a las interferencias perjudiciales. En consecuencia, la explotación de las estaciones piratas es incompatible con las Convenciones de Telecomunicaciones y el derecho internacional público, en general, cada Estado puede y debe tomar, sobre el territorio de su jurisdicción, toda medida susceptible para frenar la actividad de las piratas. Es necesario cumplir las obligaciones internacionales y no violar ningún aspecto, ni ofrecer ninguna ayuda, bajo cualquier forma que sea, dentro de su territorio a una estación pirata (recogido en el Consejo de Administración de la UER. Ginebra 17-19 mayo 1962.)

$2^{\mathrm{a}}$ ¿Estas medidas pueden aplicarse igualmente en contra de las piratas bajo pabellón de un Estado ligado por las Convenciones de la UIT?

La Comisión estimó que la cuestión del pabellón no tiene importancia jurídica, dado que la estación operaba en condiciones contrarias al derecho de gentes (Consejo de Administración de la UER. Ginebra 17-19 mayo 1962). 
$3^{\mathrm{a}}$ ¿Qué forma deberá tomar la acción del Consejo de Europa?

Para algunos bastaría que el Consejo de Europa redacte los principios que deberán inspirar las legislaciones nacionales. Para otros, esta forma de acción sería insuficiente. El Consejo de Europa deberá redactar una Convención susceptible de unir a todos los estados miembros. Pero, en este último caso ¿la Convención será aplicable solamente a situaciones internacionales o igualmente a las situaciones nacionales? Sin duda, con las piratas es imposible hacer una distinción entre situaciones nacionales e internacionales. En consecuencia, la asistencia aportada a los piratas sobre territorio nacional que sea hecha por nacionales o extranjeros, es siempre una situación nacional como prueba toda la legislación penal que no diferencia la nacionalidad del infractor. En definitiva, la Comisión está de acuerdo para pensar que la forma de acción para comprometer al Consejo de Europa no será sino la creación de una comisión en si mismo. La Comisión terminó recomendando a los organismos miembros que comuniquen a sus países miembros del Consejo de Europa, a fin que las delegaciones gubernamentales en el Comité de Expertos del Consejo de Europa tomen las medidas de cooperación más activas para recomendar que el Comité pueda informar la Comité de Ministros del Consejo de Europa.

Desde las primeras emisiones piratas comerciales, pareció evidente que, por encontrarse los equipos transmisores en aguas no territoriales, a bordo de buques instalados en torres, fuertes o plataformas sustentadas sobre suelo abandonado o apátrida, poco podrían hacer las legislaciones nacionales para suprimirlas. La única solución posible debía ser buscada en el seno de las uniones internacionales. Así fue como, a través de la UIT y de la CEPT, por cuanto forman parte de ellas las Administraciones explotadoras de los servicios de telecomunicación, se establecieron preceptos reglamentarios y adoptadas medidas conducentes al restablecimiento de la legalidad de este nuevo aspecto de las telecomunicaciones. No obstante, ante 
infractores conscientes e intencionados, con lucrativos objetivos, situados fuera de los territorios de los países miembros de la UIT y de la CEPT, como ocurre con las aguas internacionales, el significado de las recomendaciones de sus comités, las resoluciones de sus respectivas conferencias plenarias y las normas del Reglamento de Radiocomunicaciones, anejo al Convenio Internacional de Telecomunicaciones, no era más trascendente que una declaración de principios.

Planteada esta ineficacia de los preceptos ante los gobiernos de las naciones afectadas por la actividad de las primeras emisiones piratas, la UIT, impulsada por la UER y por las autoridades de telecomunicación de aquellos países, solicitó del Consejo de Europa, en 1961, la posibilidad de suscribir un convenio por el que sus signatarios se obligaran a reconocer en sus respectivas legislaciones nacionales y hacerlas respetar cuantas prohibiciones, derechos y obligaciones fueran comunitariamente adoptados. El Consejo aceptó el compromiso y designó un Comité de expertos para el estudio del problema y la elaboración de una propuesta de solución. Un año después, el Comité concluyó su primer ciclo de trabajos, del que resultó un proyecto de "Acuerdo Europeo para la represión de estaciones piratas", que fue enviado a los Estados miembros. Los gobiernos belga, británico, francés y danés sugirieron algunas modificaciones y ello obligó al Comité a redactar un nuevo texto que, en primer lugar, evitara la generalización extensiva de las figuras delictivas de explotación radiofónica pirata y, en segundo lugar, limitara las facultades represivas de los Estados signatarios a sólo cuando se dieran determinadas circunstancias en razón del territorio, nacionalidad o domicilio de los infractores y del destino de las emisiones. Se pretendía tipificar los delitos y evitar toda represión indiscriminada o anárquica; no se trataba de crear un gobierno-gendarme frente a todas o algunas de las infracciones cometidas en la materia, sino de asignar a cada país firmante del acuerdo sus exclusivas competencias y establecer un verdadero sistema de Derecho. 
El segundo ciclo de trabajos del Comité de expertos concluyó con la redacción de un nuevo texto de "Acuerdo Europeo para la represión de las emisiones de radiodifusión efectuadas por estaciones situadas fuera de los territorios nacionales", que fue aprobado, no sin dificultades, por delegados de los ministros miembros del Consejo de Europa en diciembre de 1964.

El Acuerdo consta de un preámbulo y de trece artículos. El primero remonta sus motivos a la prohibición de establecer y utilizar estaciones de radiodifusión a bordo de buques, aeronaves, o de otros objetos flotantes o aerotransportados situados fuera de los territorios nacionales, según dispone el Reglamento de radiocomunicaciones; a la utilidad de extender aquella prohibición a estaciones del mismo carácter instaladas sobre objetos fijados en el fondo del mar, o con apoyo en el mismo, fuera de los territorios nacionales, y al interés de una colaboración europea en la materia. El articulado:

1.- Define el objeto del Acuerdo como se puede leer en el artículo 1 del reglamento de radiocomunicaciones: "El presente Acuerdo contempla las estaciones de radiodifusión -y de televisión, se entiende- instaladas o en servicio a bordo de un buque, de una aeronave, o de todo otro objeto flotante o aerotransportado, y que, fuera de los territorios nacionales, transmiten emisiones destinadas a ser recibidas, o susceptibles de ser recibidas, en todo o en parte, en el territorio de una de las Partes Contratantes, o que causen una perturbación perjudicial a un servicio de radiocomunicaciones explotado con la autorización de una de las Partes Contratantes, conforme al Reglamento de Radiocomunicaciones".

2.- Determina las obligaciones (en al artículo 2 del mencionado reglamento) que adquieren los Estados signatarios para: 
a) reprimir, como infracción criminal, el establecimiento de estaciones piratas y los siguientes actos de colaboración con ellas cometidos a sabiendas:

- el suministro, el mantenimiento o la reparación de material;

- el suministro de aprovisionamiento;

- el suministro de medios de transporte o el transporte de personas, de material o de aprovisionamiento;

- el suministro de servicios referentes a la publicidad en favor de las estaciones interesadas.

b) poner en aplicación, de conformidad con sus leyes nacionales, las normas del Acuerdo con respecto a sus súbditos y a los extranjeros que, en su territorio o a bordo de sus buques o aeronaves o en los mismos medios o a bordo de otros objetos flotantes, aerotransportados o fijados en el fondo del mar o con apoyo en el mismo, hubiesen cometido los actos tipificados como delitos en el apartado anterior (arts. $3^{\circ}$ y $4^{\mathrm{o}}$ del Reglamento de Radiocomunicaciones)

3.- A los efectos de las normas anteriores no se consideran delictivos aquellos mismos actos cuando son ejecutados para socorrer naves, aeronaves, objetos flotantes o aerotransportados que se hallaren en peligro o para proteger la vida humana (art.6)

4.- Considera lícita la no aplicación del Acuerdo a las prestaciones efectuadas fuera de las estaciones de referencia (art.5)

El Acuerdo fue finalmente firmado en Estrasburgo el 22 enero 1965, sin reserva alguna por Dinamarca y a reserva de ratificación o de aceptación por Bélgica, 
Francia, Grecia, Luxemburgo, Reino Unido y Suecia, y posteriormente, aunque dentro del año 1965, por Italia, Noruega, Irlanda, Países Bajos y la República Federal Alemana (ver Nota 2). A tenor de lo dispuesto en el artículo 9, el Acuerdo entró en vigor para cada Estado miembro un mes después de la fecha del depósito, en la Secretaría General, del correspondiente instrumento de ratificación o de aceptación (ver Nota 3), permitiéndose, además, a todo miembro o asociado de la UIT no perteneciente al Consejo de Europa, previa autorización de su Consejo de Ministros, su adhesión al compromiso.

Una intensa actividad diplomática fue desplegada entonces, puesto que de nada servía el acuerdo si no era ratificado por todos y cada uno de los miembros del Consejo de Europa - lo que suponía su lenta aprobación por los respectivos parlamentos y su promulgación en cada país con rango de ley - y se lograba la adhesión de otros Estados europeos miembros de la UIT pero no del Consejo, que podían ser decisivos, al menos en el terreno de las posibilidades, como sede de las empresas explotadoras de las estaciones piratas o para el suministro de materiales, provisiones y programas de las emisoras extraterritoriales. Nuestro país, con amplias costas y numerosos puertos no muy alejados del Canal y una extendida y numerosa radiodifusión no explotada por el Estado, fue uno de los más considerados para los gobiernos promotores del Acuerdo de Estrasburgo.

En 1964 el gobierno británico inicia un pleito con R. Caroline, que concluirá en 1980 con el hundimiento del buque por orden judicial, enmudeciendo así la más famosa y longeva de las estaciones piratas. RODRIGO (1981:268)

Las características de las estaciones de radiodifusión en alta mar en servicio, según el Consejo de Administración de la UER a 1 de febrero de 1965 era la siguiente: 
Característica Frecuencia en $\mathrm{kHz}$

de la estación

Ondas largas y medias

"R. City"

1034

1625

“R. London"

"R. King"

"R. Caroline "Sud"

"R. Caroline "Nord"

“R. Veronica"
Fecha de la

puesta en servicio
Emplazamiento

aproximado
1495

1520

1562

1129,5

Diciembre 1964 Al sureste de Inglaterra

Agosto 1964 En una torre del estuario del

Támesis

Marzo $1964 \quad$ Al sureste de Inglaterra

Mayo 1964 Al noreste de la isla de Man

Abril 1960 A lo largo de las costas de los

Países Bajos a la altura de La Haya

Ondas métricas

"R. Syd"

88300

Septiembre 1961

En el Sund, entre

Dinamarca y Suecia

Figura 1: elaboración propia 
Entre septiembre de 1965 y abril de 1966 se produjeron los siguientes cambios (Consejo de Administración de la UER. Dubrovnik 3 al 6 de junio de 1966.): las emisiones de "R, City" en $730 \mathrm{kHz}$ cesaron a fin de agosto de 1965, pero las emisiones en 1034 continuaron, aumentando la potencia de la estación al final de 1965.

"R.King", que emitía en 1259 kHz, cesó sus emisiones al comienzo de octubre y fue reemplazada por un emisor más potente, en $773 \mathrm{kHz}$, denominado “R. 390”, que se estableció como “R. King” en una torre en el estuario del Támesis.

El 22 de octubre apareció una nueva estación pirata, "R. Tower", situada en otra torre del citado estuario. Cambió a menudo de frecuencia pero la más habitual es la de $1260 \mathrm{kHz}$, aunque sus emisiones cesaron a final de diciembre, pero se reanudaron del 5 al 24 de marzo sobre una frecuencia variable de 1270 a $1260 \mathrm{kHz}$, y desde el 15 de abril en $1264 \mathrm{kHz}$. A principios de diciembre anunció que difundirá espacios de televisión en el canal B5 de la Banda I, aunque algunas informaciones de prensa nos hicieron saber que el proyecto fue abandonado.

El 28 de octubre entró en funcionamiento otra estación sobre una torre en el estuario del Támesis, “R. Essex” que emitió sucesivamente en 1351, 1353 y 1349 kHz.

El 1 de enero de 1966 apreció otra nueva estación que operaba sobre las islas británicas: "R. Scotland", situada sobre un barco en el estuario del Forth en Escocia. Transmitió en 1241 para luego cambiar sucesivamente a 1237, 1231, 1255, 1257 y 1254 y 1262 y 1259 kHz el 15 de abril. Su potencia de 20kW.

El 20 de enero el barco "Mi Amigo", a bordo del cual estaba instalada "R. Caroline Sud" que emitía en $1495 \mathrm{kHz}$, fue atacado por una tempestad y embarrancado cerca 
de Friton, en la costa sudeste de Inglaterra, pero prosiguió a un puerto holandés donde se efectuaron las reparaciones necesarias.

Desde el mes de marzo, la prensa británica se preguntaba por la próxima entrada de una nueva estación "R. Ship Ocean 270" estacionada en Scarborough, (Yorkshire). La estación debería comenzar a emitir el 1 de abril, pero el barco sobre el que se encontraba fue atacado por una tempestad y se tuvo que refugiar en puerto. La tempestad originó daños a otros barcos sobre los que había estaciones piratas, especialmente a "R. Essex". Se anunció también un nuevo barco, "Olga Patricia” que ancló en las proximidades de "R. London" y "R. Caroline Sud" para emitir otro programa comercial ("R. England”).

Como curiosidad digamos que comenzó sus emisiones una estación pirata de televisión a bordo del barco "Cheeta" anclado en el Sund, a lo largo de Malmoe, como ya se dijo en el caso de "R. Syd" que la explota. Utiliza el mismo barco desde hace cuatro años para difundir las emisiones de radiodifusión en ondas métricas en 88,3 MHz, con una potencia de 20 kW. La nueva estación de televisión comenzó sus emisiones a comienzos de diciembre en el canal 41 con una potencia radiada aparente de $500 \mathrm{w}$. la antena está situada a una altura de 30 metros..

Las características de las estaciones de radiodifusión en alta mar en servicio a 20 de abril de 1966 era la siguiente, según el Consejo de Administración de la UER. Dubrovnik 3 al 6 de junio de 1966.

$\begin{array}{lccc}\text { Característica } & \text { Frecuencia en KHZ } & \text { Fecha de la } & \text { Emplazamiento } \\ \text { de la estación } & & \text { puesta en servicio } & \text { aproximado }\end{array}$

Onda largas y medias 
“R. 390"

773

del Támesis

"R. City"

1034

del Támesis

"R. London"

sureste de Inglaterra

"R. Scotland" 1259

estuario del Forth

"R. Tower" 1264

del Támesis

"R. Essex"

del Támesis

"R. Caroline "Sud" 1493

1169

"R. Caroline "Nord" 1520 “R. Veronica” 1562

Septiembre 1965 En una torre en el estuario

Mayo 1964

En una torre en el estuario

Diciembre 1964 En el barco "Galaxy", al Enero 1966

Sobre un barco en el Octubre 1965 En una torre en el estuario Octubre 1965 En una torre en el estuario Mayo 1964

Abril 1966

Sobre el barco "Cheeta 2" al sureste de Inglaterra Sobre el barco "Mi Amigo" al sudeste de Inglaterra Marzo 1964 Sobre el barco "Caroline" al nordeste de la isla de Man Abril 1960 Sobre el barco "Veronica a lo largo de las costas de los Países Bajos, a la altura de La Haya 
Ondas métricas

“R. Syd" $88300 \quad$ Septiembre 1961

Canal 41 de televisión Diciembre 1965 Sobre el barco "Cheeta", en el

Sund, a lo largo de Malmoe

Figura 2: elaboración propia

El Canal de la Mancha continuó siendo cobijo de los piratas de las ondas radioeléctricas, animados por la lentitud de las ratificaciones o aceptaciones nacionales del pacto. A mediados de 1966, año y medio después del formal acto de la firma de Estrasburgo, todavía no se había producido ni una sola ratificación. El 20 de enero de 1966, una fuerte tormenta rompió amarras del buque "Mi Amigo", nodriza de "R. Caroline South", encallándolo cerca de Frinton, en la costa sudeste de Inglaterra, a tres millas y media de su acostumbrado lugar de fondeo. Ronan O'Railhy, irlandés de nacimiento y con lujosas oficinas en el elegante Mayfair de Londres (la sede se hallaba en el mercantilmente muy internacionalizado Liechtenstein), un Francis Drake muy de nuestro tiempo, "rey de la música pop" y propietario de las dos "R. Caroline", hizo cuanto le fue posible por salvar el viejo casco y sus equipos radioeléctricos y de navegación. Cinco millones de jóvenes radioescuchas de Inglaterra y Escocia y algunos millares del continente, suspiraron con tristeza. La enorme voluntad, el empuje y el capital movilizado por O'Railhy y por su asociado Allan Crawford, propietario del buque y de su transmisor y asociado al irlandés en la explotación de "R. Caroline South", pudieron más que la mala mar y los muchos años del buque; "Mi Amigo" fue salvado y remolcado hasta un puerto holandés en donde fue reparado. En abril volvió a la mar, ahora acompañado por su reserva y sustituto "Cheeta 2", que repetía el mismo programa en otra frecuencia. La 
abundante publicidad contratada en la oficina de Mayfair y el ya numeroso personal de la empresa no permitía una larga interrupción del servicio.

Fruto de la intensa actividad del Reino Unido para contrarrestar la influencia de estas estaciones en 1967 empieza a emitir la primera estación local de la BBC, en Leicester, al tiempo que se prohíben las estaciones piratas, RODRIGO (1981:269)

En aquella época hubo nuevas altas en la colonia corsaria del Canal: "R. Ship Ocean 270" (abril 1966), anclada a cuatro millas de la costa de Scarborough, Yorkhsire, al norte de Inglaterra, y "R. England" (junio 1966), instalada en el "Olga Patricia", fondeado junto a "R. London", ante Felixtowe, a cincuenta o sesenta millas de Londres. "R. England" - se escribió en la prensa al tiempo de su aparición - contaba con "el apoyo financiero de una empresa de Tejas en la que tiene intereses la esposa del Presidente Johnson" LABORDE (1967). También hubo bajas: "R. King" (ex-"R. Invicta"), y "R. 390", ambas inquilinas de los fortines montados sobre las "Red Sands". El juez O'Connor, del Tribunal Supremo, resolvió un recurso de la Administración contra los representantes de "R. 390", definiendo que aquellas arenas, descubiertas de agua en algunas mareas, eran tierra de la Gran Bretaña. En consecuencia, la policía judicial tomó nueva posesión de las casamatas montadas sobre pilotes y selló las instalaciones; ROBBINS (1967:81-82)

Por entonces, las tarifas publicitarias de las emisoras piratas del Canal, dirigidas al mercado inglés, eran las siguientes: 
Emisoras piratas Mercado Tarifa por minuto (libras) Tarifas por minuto (pesetas)

"Caroline South" Londres $\quad 180$

28800

"Caroline North" Costa NW (nota 4) 180

28800

"Radio London" Londres

152

24320

"Radio England" Londres

152

24320

"Radio City" $\quad$ Londres

80

12800

"Radio Ocean 270" Costa NE

60

9600

"Radio Scotland" Escocia (nota 5) 56

8960

"Radio Essex" $\quad$ Londres

20

3200

Figura 3: elaboración propia

"R. Veronica", incidiendo principalmente sobre Holanda y en algunos programas sobre Inglaterra y Bélgica y "R. Syd", en FM, todavía en el Sund, completaban la plantilla de estaciones piratas del verano de 1967, cuando el Parlamento británico, como consecuencia de su ratificación del Acuerdo Estrasburgo, aprobó una ley por la que se prohibía el funcionamiento de toda emisora de radiodifusión que operase en frecuencias no registradas. Esta acción, acatada por todas las estaciones piratas menos por las dos Carolines, coincidente con la ratificación por los países nórdicos 
del pacto represivo, modificó sensiblemente el panorama de la radiodifusión pirata durante los años siguientes.

En marzo de 1968 solo "R. Véronica" subsistía en la integridad del panorama de la onda media europea, según Informe de la Comisión Técnica de la UER a su Asamblea General, reunida en La Haya, del 5 al 8 de julio de 1968 . Con tal motivo, el Consejo de Administración de la UER remitió una carta a la dirección general de los PTT holandeses en demanda de una rápida ratificación del Acuerdo por los Países Bajos. Entre otros sensibles perjuicios, "R. Veronica" interfería la red sincronizada de la radiodifusión sueca (SR), que operaba en 1562 kHz, su misma frecuencia

Pero el Gobierno y el Parlamento holandeses no estaban dispuestos, por entonces, a renunciar a su liberalismo filosófico ni a enfrentarse a una juventud permanentemente contestataria y ferviente partidaria de la emisora situada frente a Scheveningen. Todo lo que podía hacer - y lo hizo - fue recomendar a la Fundación Holandesa de Difusión, NOS, empresa oficial de la radiotelevisión, la realización de emisiones de la música preferida de la audiencia juvenil para minar las bases de sustentación de "R. Véronica". Por ellos salió al aire el programa "Hilversum 3": música "pop" las veinticuatro horas, sin publicidad. Bob Dylan, Eddie Cochrane, los Beatles y los Rolling Stone llenaron el espacio, ahora servidos por una corporación de carácter público (La "Nederlandse Omroep Stichung", NOS). Ésta fue una fundación creada por ley que coordinaba los programas de las asociaciones productoras, supervisaba la dotación de los estudios y era la responsable de los servicios técnicos comunes para la difusión de los programas.

A causa de la inmovilidad de las autoridades holandesas, del éxito económico de "R. Veronica" y pese a los esfuerzos de la CEPT (Recomendación TR/10 (1971) del Grupo de Trabajo T/TG 3, "Radiocomunicaciones" de la Comisión "Telecomunicaciones) y 
de las Conferencias de Ministros Europeos de Correos y Telecomunicaciones (Conferencias de Bruselas ,23-28 abril 1970 y Viena 11-12 abril 1972)., volvieron a salir de sus guaridas dos viejas estaciones piratas ("R. Amigo" y las Caroline North y South, ahora fundidas en una sólo, "R. Caroline", que operaba frente a la costa del Sudeste de Inglaterra), mientras entraron otras dos ("R. Atlantis" y "R. North Sea International") en funcionamiento. Ante los riesgos y el agravamiento de la situación, la UER y los países comprometidos en Estrasburgo a suprimir estas emisiones volvieron a presionar sobre el gobierno holandés, el cuál, al fin, decidió enviar a las Cámaras el Acuerdo de Estrasburgo para su ratificación. El entonces Primer Ministro, B. Biesheuvel, en febrero de 1973, solicitó de la Segunda Cámara la desaparición de las estaciones piratas situadas frente a las costas holandesas por perturbar la radioescucha marítima y la radiodifusión normal.

Anunciado uno de los debates parlamentarios para el 18 de abril, unos cien mil jóvenes, apasionados y habituales escuchas de "R. Veronica", paralizaron, pacífica y alegremente, el centro de La Haya para concentrarse en Malieveld, a escuchar un concierto de música "pop" y marchar hacia el Binnenhof, sede de los edificios de las Cámaras. Una comisión de manifestantes hizo llegar a los diputados una petición escrita de continuidad de las emisiones de su estación preferida, apoyada por dos millones de firmas. No obstante la demanda, la Segunda Cámara aprobó la ratificación del Convenio el 28 de junio de 1973 lo que ocasionó otra manifestación, esta vez de sólo mil manifestantes pero muy violenta, de verdadero enfrentamiento entre la policía y los simpatizantes de "R. Veronica", quienes lanzaban piedras y botellas y atacaban a los agentes con cadenas de bicicleta. El Acuerdo pasó a la Cámara Alta en donde permaneció un año más.

Hagamos un paréntesis para poner de manifiesto que este movimiento no solo se producía en Europa sino en otros países. 
Así en 1966, comienza a emitir la emisora alternativa KMPX de San Francisco (Estados Unidos), bajo la dirección de Tom Donahue, sirviendo de portavoz del movimiento de protesta por la intervención armada norteamericana en Vietnam. Sucede en un momento en que las FM han hecho resurgir la audiencia de las emisoras de radio con programas únicos de formato continuo, dirigidos a un sector muy determinado del público creando, además, un nuevo estilo radiofónico, basado esencialmente en la figura y modo de hacer del disk-jockey, que tendrá una influencia decisiva en el posterior desarrollo de la radiodifusión alternativa, RODRIGO (1981:268-269)

A principios de 1974 la situación era la siguiente:

\section{Estaciones piratas}

- "R. Veronica" a bordo del "Nordeney", continuaba emitiendo frente a Scheveningen, en nueva frecuencia de $557 \mathrm{kHz}$.

- "R. Atlantis", a bordo del Janine", surto en el Mar del Norte, en onda de 1313 kHz.

- "R. Caroline", a bordo del "Mi Amigo", también en el Mar del Norte, en onda de $1187 \mathrm{kHz}$.

- "R. Mi Amigo", en el mismo buque que la anterior, igualmente en 1187 kHz. El mismo transmisor operaba desde seis de la mañana a siete de la tarde como "Mi Amigo", en lengua holandesa y el resto del tiempo, hasta las seis de la mañana del día siguiente, como "R. Caroline", en lengua inglesa. 
- "R. North Sea International", a bordo del "Mebo-2", sin matrícula, fondeado también en el Mar del Norte y emitiendo en frecuencias de 1367 kHz, 6210 kHz y 101 MHz.

\section{Acuerdo de Estrasburgo}

- Ratificado por todos los signatarios con excepción de Italia, Suiza y Países Bajos.

- Solicitada, por la vía diplomática, la aceptación de Austria, miembro del Consejo de Europa, y la adhesión de España.

Después de merecer la aprobación de la Cámara Alta de los Países Bajos, la ley de 27 de agosto de 1974, que entró en vigor en 1 de septiembre, ratificó el Acuerdo de Estrasburgo y las modificaciones precisas de la ley de Telecomunicaciones para configurar los delitos y las obligaciones del Estado resultantes de la aplicación de aquel pacto internacional.

Las estaciones piratas - menos "R. Caroline" y "R. Mi Amigo" - dejaron de funcionar. Los buques abandonaron sus fondeos para acudir a puertos seguros de los que ya no volverían a salir. "Mi Amigo", como caso aislado, salió de las aguas vecinas al mar territorial holandés para situarse cerca del buque-faro Kentish Knock, frente a la costa de Essex.

Las autoridades holandesas se vieron en parte frustradas. "R. Veronica" - la estación que había obtenido veinte millones de florines anuales de beneficios - había desaparecido, pero "R. Mi Amigo" cubrió con creces el vacío, además de mantener su antigua clientela de los Países Bajos. Por su parte, "R. Caroline" continuaba fijando la atención de la escucha inglesa. Se intentó entonces, en el ambiente político y en la prensa holandeses, desenmascarar los intereses que se ocultaban detrás de "Mi 
Amigo" con su potente transmisor de $50 \mathrm{Kw}$ de potencia y antena de $60 \mathrm{~m}$. de altura, capaz de cubrir una zona circular de $500 \mathrm{~km}$. de radio.

Para resolver la presencia de firmas holandesas en la publicidad de la emisora, se adoptó una fórmula que curiosamente elude la prohibición de contratar anuncios a emisoras piratas: las empresas ordenan textos publicitarios en la revista "Joepie" a coste de tiempo de lectura; esto es, al tiempo precisado para leer un anuncio por un locutor y al precio de 12,50 florines (315'60 pts.) por segundo. Estos anuncios se publican en "Joepie" y son leídos por los locutores de "R. Mi Amigo". La justicia holandesa, encargada de aplicar las disposiciones del Acuerdo de Estrasburgo y la ley de Telecomunicaciones, no pudo evitar que las firmas holandesas contraten en Holanda publicidad para una revista holandesa, y tampoco puede considerar como ilícito que un locutor de una emisora pirata lea los anuncios de una revista musical. Ello no impide que las marinas inglesa y de los Países Bajos vigilen de cerca los buques que aprovisionan al "Mi Amigo": "Causa causae est causa causanti".

Un año antes, como se dijo, la NOS montó una nueva emisora, la "Hillversum 3", para disputar la audiencia de "R. Veronica" que transmitía música moderna, aunque sin publicidad, y que iba ganando en popularidad. Entre 1965 y 1971 pasó del 4,9 al 10\% en tanto que "R. Verónica" disminuyó del 8,8 al 4,4\% en el mismo período, según nota de 9 de febrero de 1973 del Consejero de Información y Turismo (Luis Escobar de la Serna) al Subsecretario de información y Turismo, donde confirma que las tres estaciones piratas situadas fuera de las aguas territoriales de Holanda: "R. Verónica”, “R. North Sea International” y “R. Caroline”.

PAULU (1968:21) cifra en 200.000 libras (560.000 dólares) el coste de una estación de onda media, montada sobre un viejo pesquero de tonelaje medio o pequeño mercante; una plataforma podía costar el doble; el arreglo de un fortín abandonado o 
plataforma de radar y artillería antiaérea, como los del estuario del Támesis, valdría no más allá de cien mil libras. Sin embargo, los beneficios líquidos mensuales, en todos los casos, podrían ascender a 80.000 libras, 224.000 dólares lo que significa la amortización total de las inversiones cada dos meses y medio o, en otras palabras, unos rendimientos mensuales del $40 \%$ equivalentes al $480 \%$ al año. Pocos negocios iguales. Incluso algunas emisoras, como "R. Veronica", duplicaron estos increíbles beneficios, no sujetos a tributación alguna, ni a seguridad social de su personal.

Se crea una nueva estación pirata en la zona del Mar del Norte. Dicha estación emitirá desde el barco "Elizabeth" propiedad de W. Beusenberg, hombre de negocios relacionados con las pesquerías. Se desconocía la frecuencia. Esta estación hará el número cuatro entre las que están situadas fuera del límite de las aguas territoriales holandesas.

Meses más tarde, apareció en Holanda "R. Condor", en 270 metros, con el deseo de emitir música ligera con mensajes humanitarios, sociales y religiosos. Su representante en tierra era la Sra. Van Donselaar, ama de casa que declaró que con un grupo de amigos idealistas y los ahorros de dos años y medio habían concluido una estación de $500 \mathrm{w}$, instalada en el barco "Condor", frente a la playa de Zandvoort. Los propietarios del barco eran S. Willemse, técnico de radio y televisión, y Gerrit Elfering, director ejecutivo de una compañía de salvamento. Todos vivían en Haarlem.

Por su parte, la NOS, había solicitado al Parlamento holandés la ratificación del Acuerdo de Estrasburgo, dado que la estación "R. Veronica" había reanudado sus emisiones desde el buque de su principal competidor "R. Caroline" y habían firmado un acuerdo por un mes para emitir sus programas mientras se celebran las sesiones del Parlamento. 
Los propietarios de "R. Veronica", cuyo buque encalló en la costa a consecuencia de un temporal, apelaron a 250.000 personas para que realizasen una marcha demostrativa ante el edificio de la Segunda Cámara, llevando receptores portátiles sintonizados con "R. Veronica”. La manifestación formaba parte de una campaña en favor de dicha estación, que también incluía un plan para adornar el edificio de la Segunda Cámara con flores y hacer sobrevolar aviones por el centro de la Haya, llevando banderas con slogans de apoyo a "R. Verónica".

El Senado holandés discutió las llamadas "pequeñas leyes Veronica", que, en junio de 1973, fueron aprobadas por la Segunda Cámara y que acarrearán la desaparición de las tres estaciones piratas: “R. Verónica”, "R. Caroline” y “R. Sea International y otras similares situadas en el Mar del Norte, fuera de las aguas territoriales.

El Ministro holandés de Cultura, Van Dorn, decidirá sobre la solicitud de espacio de emisión dentro del sistema radiofónico de Hilversum, presentada por la recién fundada "Organización de Difusión Verónica" (VOO). El Ministro hizo esta promesa sobre todo para satisfacer a la fracción del partido católico, puesto que dependía en gran medida de esta fracción si el Gobierno hubiese obtenido la semana siguiente la mayoría de los votos.

Gran parte de la primera Cámara expresó severas críticas contra el Ministro Van Doorn, puesto que hasta ese momento no había hecho nada para cubrir el "vacío" que se originaría con la desaparición de "R. Verónica". Los partidos liberal (VDD), cristiano-histórico (CHU), D’66 y una minoría de la fracción católica, señalaron que existía una gran necesidad de una estación "pop". Un diputado del grupo D'66 propuso hacer entrar en vigor la prohibición de las estaciones piratas en octubre. Los partidos cristiano-histórico, liberal y pacifista apoyaron esta propuesta. 
El antiguo Ministro de justicia del partido liberal estimó injustos los ataques contra "R. Verónica", puesto que únicamente ha hecho uso de una laguna existente en la ley.

El Secretario de Estado de Tráfico dijo creer que Holanda tendría una mala posición en la conferencia sobre la distribución de ondas, si en esa fecha no hubieran desaparecido todas las estaciones piratas.

La propuesta se llevo a cabo y existe legalmente la estación de radiodifusión y de televisión “R. Verónica” (VOO).

Holanda ratificó el Acuerdo de Estrasburgo el 27 de agosto de 1974 y entrará en vigor el 1 de septiembre de 1974. En consecuencia, el 31 de agosto de 1974 a las 19.00 horas GMT “R. North Sea International” dejó de emitir en la frecuencia de 1367, $6210 \mathrm{kHz}$ y 100,8 MHz. El barco “Mebo 2", desde el que se transmitía, se quedó en el puerto de Rotterdam por razones de mantenimiento. De acuerdo con una información extraoficial, el barco navegó a vela por el mediterráneo para empezar a emitir en las lenguas inglesa e italiana desde la costa de Italia.

“R. Verónica” (557kHz) cesó en sus emisiones el 31 de agosto a las 18.00 horas GMT. Como refleja el Telex del Grupo de Trabajo Radiocomunicaciones de la CEPT, fechado el 24 de septiembre de 1974.

El barco "Nordeney" está todavía fuera de aguas territoriales en Scheveningen. Como reflejaba el Telex del Grupo de Trabajo Radiocomunicaciones de la CEPT, fechado el 24 de septiembre de 1974. 
"R. Atlantis" (1313 kHz) también dejó de emitir el 31 de agosto a las 18.00 horas GMT. Sobre el barco "Janine" recayó un embargo. En dicho barco se encontraba la instalación desde la que se transmitía. En ese momento el barco estaba en el puerto anclado. Así los refleja el Telex del Grupo de Trabajo Radiocomunicaciones de la CEPT, fechado el 24 de septiembre de 1974.

"R. Mi Amigo "y "R. Caroline", ambas transmitiendo desde el barco "Mi Amigo", continuaron sus retransmisiones en la frecuencia $1187 \mathrm{kHz}$. Las estaciones continuaron en el aire con un programa en holandés. Durante la tarde y la noche, dado que "R. Caroline" tenía un programa en inglés. El barco "Mi Amigo" había cambiado su situación y ahora estaba situado en la costa inglesa cerca de Felixstone. De acuerdo con una información extraoficial, el barco fue expulsado de los puertos españoles.

En numerosas reuniones de la CEPT se trató el tema para estudiar la forma de reprimir estas estaciones piratas, ya que no solo afectaban a las bandas de radiodifusión sonora sino a las de navegación radiomarítima.

El Gobierno de Holanda autorizó a la Verónica Broadcasting Organisation con la categoría C (nota 6).

El Centro Técnico de la UER afirma en una circular de 22 de mayo de 1978 que en el mar del Norte continua "R. Mi Amigo/R. Caroline" en 962 kHz. "R. Mi Amigo" emite por la mañana un programa en holandés y "R. Caroline" por las tardes noches en inglés. En el Mediterráneo oriental emite "R. Paix" en 1538 kHz captada en Jurbisa, Caversham, Sorrento y Tel Aviv. Ciertas informaciones afirman que ensaya la emisión en $6250 \mathrm{kHz}$. La estación "Mebo II" se capta esporádicamente en una frecuencia variable de 6204 a $6206 \mathrm{kHz}$ especialmente en Jurbise. 
Como consecuencia de la presión española, “R. Caroline” interrumpió sus emisiones el 23 de enero de 1979 (nota 7) (según se puede leer en el periódico "Le Monde" 23 janvier 1979 p. 31). Sin embargo, como veremos en el apartado siguiente, la estación reanudó sus emisiones.

El 20 de marzo de 1980, el barco que acogía a "R.Caroline", debido a una fuerte galerna, se hundió (nota 8). Pero reanudó sus emisiones, en 319 m., en un bou (nota 9), según la prensa española (Cinco días y El País 04/08/1983), en un buque de 976 toneladas acondicionado en un astillero español y rebautizado como "Imagine" estaría anclado a unos $20 \mathrm{~km}$ de la costa inglesa. La nueva estación cuenta con una ayuda de 600.00 millones de pesetas, procedentes de inversiones norteamericanas.

En octubre de 1984, el gobierno inglés lanzó una ofensiva con el fin de clausurar las más de 50 estaciones privadas instaladas en territorio británico. El Ministro de Industria, responsable de la vigilancia de las ondas, se manifestó especialmente en contra de las estaciones piratas extranjeras, como las de las comunidades turca y griega.

El gobierno británico culminó, en 1985, una serie de acciones que estaba llevando a cabo en su lucha contra las estaciones piratas situadas en su territorio o en su área de influencia. A principios de febrero, las autoridades confiscaron los transmisores de seis estaciones que operaban ilegalmente en Londres, entre ellas R. Jackie, la más estación pirata más antigua del país, creada en 1969 y que emitía las 24 horas con una audiencia en torno a los 20.000 oyentes. Se encontraba situada en Worcester Park.

El gobierno británico lanzó una gran ofensiva contra dos estaciones piratas (como recogía Diario 16 el día 16 de Octubre de 1985) “R.Laser” y “R. Caroline”, alquilando 
un barco para controlar todos los buques que se acercaban para abastecer de alimentos y combustible e identificar a los responsables para detenerlos y procesarlos. Cualquier individuo que trabajase para esas estaciones podía ser multado con más de 400.000 pesetas o condenado a dos años de prisión, según la ley de Emisiones marinas de 1957. Pocos meses más tarde una tormenta acabó con la estación "R. Laser" que tenía una audiencia de cuatro millones de personas en Gran Bretaña sólo. “R. Carolina continuó hasta 1995, o sea emitió durante treinta y tres años. Ahora lo hace por satélite, pero esa es otra historia.

\section{Conclusiones}

En el marco de los convenios y de los reglamentos internacionales vigentes, la radiodifusión pirata es ilegal por su origen, naturaleza y destino de sus programas. Sin embargo, estas emisoras han creado un novísimo estilo radiofónico, atractivo para la generalidad de los oyentes y apasionante para la audiencia juvenil. Para las estaciones piratas, el ritmo es el mensaje. Música pop muy del tiempo, publicidad dosificada hasta el máximo soportable y noticias deportivas; esta es la fórmula magistral de su programación durante las 24 horas del día, mantenida por contacto directo del locutor-presentador con su audiencia. No es un disco dedicado a un oyente; es un disco dedicado, simultáneamente a todos y cada uno de los oyentes, que llega plenamente a sus destinos en una comunicación directa e íntima. Publicidad medida: 6 minutos por hora; ni uno más, lo que haría exceder los anuncios de la capacidad receptiva del espectador medio europeo; ni uno menos, a ser posible, para no desacostumbrar el arraigo del programa comercial. En todo caso, la publicidad se presenta como el resto del programa, con el mismo ritmo, la misma presentación altamente comunicativa, la misma forma de mensaje. 
Dirigido a todos y cada uno de los oyentes como si fuera exclusivo para él. De lo deportivo, solo lo trascendental, lo que importa a los diez millones de oyentes de "R. Caroline" o a los siete millones de "R. Mi Amigo". De lo demás -guerras, epidemias, catástrofes, política, conflictos sociales- nada; nada que no sea la música que invita a moverse y el anuncio que incita a comprar.

Convengamos que la nueva tecnología ha permitido soslayar este tema de extraterritorialeidad tal como afirma el profesor David Caldevilla: "Los planteaminetos emergentes (de Internet) se basaban en una incipiente autorreferencialidad de los medios q buscaban crear un nuevo lenguaje informativo y de entretenimiento a través de un concepto que los clásicos dignificaron con la definición deixis" (CALDEVILLA DOMÍNGUEZ, David. "La radio por Internet: La comunicación del siglo XXI" en Radio y Televisión en el ámbito local. Ed. Comloc. Universidad Jaime I. Castellón. 2002)

La radiodifusión pirata es actividad de altos rendimientos económicos. Sus planteamientos son rigurosamente mercantiles. ¿Por qué la juventud europea ha vivido ajena a los medios de comunicación social de nuestro tiempo, unos medios que por definición son de masas y es ella, la juventud, la parte más numerosa de esa masa? ¿Por qué la juventud no lee los periódicos, ni oye la radio, ni ve los programas de televisión, ni asiste a los cinematógrafos, ni ve las obras de los teatros que suelen leer, ver y oir los mayores? ¿Qué caracteriza a la juventud de nuestro tiempo?. Si Ronan O'Railhy, los hermanos Verweijk y Sylvain Tack no plantearon así sus respectivos negocios es porque tienen un talento natural que suple con creces las reflexiones a que invitan aquellas preguntas. Consciente o intuitivamente supieron que la juventud actual, disconforme y contestataria propia de los países occidentales, es también una juventud rica, que dispone de dinero fácil para sus gastos aunque no 
suele gastarlo en los mismos artículos de consumo que sus mayores. Los jóvenes de hoy trabajan o pertenecen a familias que para sus gastos les dan más de lo que necesitan en sentido estricto. ¿Por qué no conducirles a gastar ese dinero en prendas de vestir especialmente hechas para ellos, en cigarrillos con paquetes de su estilo, en bebidas cuyo lanzamiento publicitario se materializa en "singles" que podrían estar cantados por los "Rollings Stone".

El gran talento de la radiodifusión pirata - y de los anunciantes que creyeron en ella como vehículo de su oferta - fue la captación de esa juventud, la gracia con que la transformó en mercado, en objeto de consumo. Florines, libras, marcos y francos, saltaron de los bolsillos de los jóvenes a las tiendas de ropas (se compran nuevas pero parecen viejas), a las cafeterías, a los puestos de venta de tabaco, a algunos contadísimos - espectáculos sobre los que flota el mismo espíritu de los programas de Veronica, Caroline y Mi Amigo, para terminar, por último, en las arcas de los explotadores de esta radiodifusión marginal pero no marginada por la sociedad de nuestro tiempo.

En 1966, las investigaciones de audiencia daban 10.500 .000 radioescuchas regulares de las emisoras piratas y 10.330.000 de la emisora central de la BBC en la zona de Londres.

Pero no olvidemos que estas estaciones en lo que respecta a música y la forma de hacer publicidad no tenían nada que envidiar a nuestras estaciones comerciales que transmitían una música buena para la época, sin hablar de los discos censurados que eran todos los extranjeros (suponemos que los censores no sabían idiomas) y algunos españoles, cuyas letras ahora nos producen risa. Como no difundían información, la equiparación era mayor y la programación volvió a mejorar con la libertad de 
información y consiguiente desaparición de la censura tanto para la música, como para la información, empezando una época de esplendor en la radio española.

En la actualidad, febrero de 2008, se han legalizado, han sido suprimidas o cerradas todas las estaciones piratas comerciales.

\section{Bibliografía}

\subsection{Tratados, Revistas, Periódicos y Acuerdos Internacionales}

“Accord Européen pour la represión des émissions de radiodifusión efectúes par des stations hors des territorios nationaux". Séries de Traités et convencións européens. No 53. Conseil de l'Europe .1965.

“Acuerdo europeo para la represión de las emisiones de radiodifusión efectuadas por estaciones situadas fuera de los territorios nacionales": Texto. Boletín Oficial de las Cortes Españolas. Núm. 1438. 6 de junio de 1975.

"Atelier de création radiophonique: radio pirates et alternatives" en France-Culture". 16 janvier 197720 h 40.

“Belgique: “Radio Plus” saisie par la police”. Liberation. 22 mars 1980.

Comisión de Telecomunicaciones: Circulares T 2/71 y AP 3/71 de la Conferencia Europea de Ministros de Correos y Telecomunicaciones (C.E.P.T.). 26 de febrero de 1971.

"Convenio sobre mar territorial". 
“Deutschland. Gesetz gegen. Piratensender gebilligt" en AV und ZV. nº 28 - 1969.

EORT (1969) "Emisoras piratas". "Life Internacional" traducido en Cuadernos de Documentación de la Escuela Oficial de Radiodifusión y Televisión. Mayo de 1969.

"De Standaard", Bruselas. 25 febrero 1975.

“El Gobierno Thatcher quiere combatir las ondas libres potenciando las munipales. Las emisoras piratas, como alternativa a la BBC" en "La Vanguardia". 18 septiembre 1985.

“European Agreement for the Prevention of Broadcasts Transmitted from Stations Outside Nacional Territories". European Trenty Series. No. 53. European Council. 1965.

"Instrumento de ratificación del Acuerdo Europeo contra las Emisiones de Radiodifusión efectuadas por Estaciones situadas fuera de los territorios nacionales, hecho en Estrasburgo el 22 de enero de 1965". Boletín Oficial del Estado no 56.5 marzo 1988.

“Irish radio. Pirates go public".en The Economist. 27 agosto 1983.

“La libertad del país más pequeño del mundo está amenazada. Las radios piratas acosan en Sealand" en El País. 26 agosto 1979.

“Las autoridades inglesas ahora ponen cerco a Radio Caroline. Una tormenta acabó con Radio Laser 558, la emisora pirata que emitía desde un barco" en Diario 16. 29 noviembre 1985. 
"Ley Federal contra las emisoras piratas".en Cuadernos de documentación EORTV. Diciembre 1972.

"Memoria de las Naciones Unidas". Consejo Económico y Social. E/Conf. 6/30 marzo 1948.

“Musica per tutti dagli studi della "Mi amigo" en Millecanali n 26. Febrero 1977.

“Netherlands: Former pirate broadcaster applies for oficial status" en EBU Technical Review. August 1977.

"Ofensiva del Gobierno inglés contra las emisoras piratas" en El Noticiero Universal", febrero 1985.

"Pirate radio. Jolly roger, over and out" en The Economist. 9 april 1983.

“Radio Caroline" en Le Monde. 23 janvier 1979.

“Radio Caroline” en Le Monde. 24 août 1983.

“Radio Caroline” en Correspondence de la Presse. 23 janvier 1979.

“Radio Caroline a coulé" en Le Monde. 22 mars 1980.

“Radio Caroline a coulé" en Le Matin. 21 mars 1980.

“Radio Caroline de nuevo en las ondas" en Cinco días. 4 agosto 1983. 
"Radio-Caroline ne se rend pas" en Le Monde Radio-Télévision. 28 de août a 3 septembre 1995.

“Radio Caroline vuelve a emitir en el Reino Unido” en El País. 4 agosto 1983.

“Radio Caroline y Radio Laser están en barcos. Gran ofensiva del Gobierno británico contra dos originales radios piratas" en Diario 16. 16 octubre 1985.

“Radio Mi Amigo. Una emisión “pirata” en Mundo diario. 3 noviembre 1978.

"Radio pirates are swarming back" en The Times. 9 august 1984.

"Radio Verónica on 1224" en página de Internet: http:// www.offshoreradio.de/1224/verabove.htm

Reglamento Radiocomunicaciones de la UIT. Ginebra. 1959.

Reunión de la C.E.P.T. Bruselas 23-28 abril 1970.

"Se fue al fondo del mar Radio Carolina" en ABC 22 marzo 1980.

"Spaanse commerciële stations spelen door naar Mi Amigo" en De Standard. 25 février 1975.

"Stations de radiodifusión en Haute mer: Naufrage du cargo portant l'emetteur de “Radio Carolina”. Revue de l’UER. Cahier Technique nº 180 avril 1980. 
"Storia delle radio pirata: radio north sea internacional" en Millecanali no 15 . Marzo 1976.

"Storia di radio pirate: radio Caroline la stazione olandese che si sente di notte in Italia" en Millecanali nº 16. Abril 1976.

“Thatcher hunde radio Jackie" en El Correo Catalán. 7 febrero 1985.Tempete. “Radio Caroline" fair naufrage au large de l'Angleterre" en Liberation. 22 mars 1980.

“Verónica is Promoted" en Intermedia. March 1979.

\subsection{Libros}

AGUILERA, Miguel (1985). Radios libres y radios piratas. Forja. Madrid.

BLACK, Peter (1972). The Biggest Aspidistra in the world. BBC. London. 1972.

BRIGGS, Asa (1961). The History of Broadcasting in the United Kingdom. Oxford University Press. London. Vol 1:"The Birth of Broadcasting". 1961. Vol 2: "The Golden Age of Wireless". 1965. Vol3: “The War of wors”. 1970

BROWNE, Don R. (1971) "The BBC and The pirates: a Phase in the Life of a Prolonged Monopoly". Journalism Quartely. Spring.

CALDEVILLA DOMÍNGUEZ, David: "La radio por Internet: La comunicación del siglo XXI" en Radio y Televisión en el ámbito local. Ed. Comloc. Universidad Jaime I. Castellón. 2002. 
COLLIN, Claude (1979). "Ecoutez la vrai difference" en La pensée sauvage. Claix. p. $11 \mathrm{y}$ sig.

COSTA, J.M. (1980). “De Radio Caroline, que emitía desde un barco sólo ha quedado un canario. Desaparece bajo el mar la última emisora pirata de música "pop". Diario El País. 22 marzo 1980.

CRIEL, Theo (1975). “Mi amigo op de spaanse radiotoer". Gazet van Antwerpen. 25 février.

GAIDO, Marco (1981). "Los orígenes: la FM, los disk-jockeys y las radios piratas". en De las ondas rojas a las radios libres. Gustavo Gili. Barcelona. p. 172.

DEBBASCH, Charles (1967). Traité de droit de la radiodifusión Ed. L.G.D.J..Paris.

DOVKANTS, Keith (1984). “Radio pirates booming” Evening Standard. 9 august.

EMERY, Walter. B.(1969) National and International Systems of Broadcasting. Michigan State University Press. 1969.

EVEN, Martin (1975). “Les malheurs d'un pirate. Revoici Caroline”. Le Monde. 21-22 decembre.

HANSSON, Gunnar (1967). “Révision de la loi suédoise concernant les stations “pirates". Revue de 1'UER. no 101 B. Genève. Janvier.

HOPKINS, Anthony. (1980) "Radio Caroline Goes Down in Force 9 Gale". Daily Telegraph. 21 march. 
IEZZI, Frank (1965). “TV Piracy on the High Seas”. Television Quartely IV. Winter.

KNOT, Hans (1999). “Rare pictures from radio's past Scandinavian Offshore Radio” en Soundscapes vol 2 avril 1999 en la página de Internet: http:/ / icce.rug.nl/soundscapes/DATABASES/RP2/Scandinavian_offshore_radio.s html

KNOT, Hans (1999). “Rare pictures from radio's past Scandinavian Offshore Radio: Radio Mercur" en Soundscapes vol 2 avril 1999 en la página de Internet: http:/ / icce.rug.nl/soundscapes/DATABASES/RP2/Mercur01.shtml

LABORDE, Enrique: (1967). “Londres: Hoy serán clausuradas las emisoras pirata”. La Vanguardia. 15 agosto.

McLEOD, Norman (1984). "The problem with pirates" en Broadcast Sound. July/august.

NAMUROIS, Albert (1965). "La répression des activités des stations pirates de radiodiffusion" en Revue de l'UER. nº 90 B. Genève. Mars.

PAULU, Burton (1968). "Radio and Television Broadcasting on the European Continent". University of Minnesota Press. Minneapolis. $2^{a}$ ed. p. 21.

PEZUELA, Alfonso (1980). “Gran Bretaña: era la última emisora pirata Se hundió Radio Caroline" en Pueblo. 21 marzo. 
PUJADES, Pius (1977). “Radio "Mi Amigo" tiene su base en Platja d'Aro. Radio pirata"legal" y única" en Tele-Expres. 30 agosto.

REMES, Karel (1965). "Répression d'emissions à partir de l'île artificielle. Loi néerlandaise sur les instalations en mer" en Revue de l'UER nº 90 B. Genève. Mars.

ROBBINS, E. C.(1967). "Déclin des pirates. La situation britannique que évolue" en Revue de l'UER. nº 106 B. Genève. Novembre.

ROBBINS, E. C.(1967b). "Le Ministre des postes et les pirates" en Revue de l’UER. nº. 102 B. Genève. mars .

ROBBINS, E. C.(1967c). "Déclin des pirates". Revue 1'UER. no. 106 B. Genève. Novembre p. 81-82.

RODRIGO, Miquel; RUIZ-COLLANTES, Xavier: (1981). “Cronología” en De las ondas rojas a las radios libres. Gustavo Gili. Barcelona. p. 268.

SEDANO LAÑO, José $\mathrm{M}^{\mathrm{a}}$ : El ayer de Radio Vitoria. spi. s/f.

SETON, Craig (1980). "Sea silences original pirate station" en The Times 21 march.

SNODDY, Raymond (1983). “Transmitter on, Alice is go..." en Financial Times 9 april.

TAMMES A. J. P. (1964). La Liberté de la Haute-mer: Légitimité d'une île de télévision" en Revue de l'UER. nº 86 B. Genève. Juillet. 
UER: diferentes documentos del Consejo de Administración y de la Asamblea General

UIT: Reglamento de Radiocomunicaciones, anejo al Convenio Internacional de Telecomunicaciones. Ed. Secretaría General de la UIT. Ginebra. 1976.

VV. AA.: (1981) De las ondas rojas a las radios libres. Gustavo Gili. Barcelona.

\section{Notas}

Nota 1: De acuerdo con los usos y acuerdos internacionales del primer período de vida de la radiodifusión, a cada licencia de emisión otorgada correspondía un indicativo, compuesto por una o dos iniciales fijas para identificar el país en cuyo territorio estaba situada la estación, otra letra para representar el código admitido por cada emisora, y un número de matrícula como registro de la concesión. A España correspondieron las iniciales EA, como distintivo, y ajustó los códigos J y R, el primero para las emisoras de cuarta categoría, es decir, emisoras de radiodifusión, y el segundo para las de quinta o de radioaficionados, de tal forma que EAJ-62 significa: "España, estación de cuarta categoría, no 62". En otras palabras, R. Vitoria. (SEDANO LAÑO, José Mª : El ayer de Radio Vitoria. spi. s/f).

Nota 2: Un análisis más detallado sobre el acuerdo puede verse en el artículo de la revista de la UER n ${ }^{\circ}$ 90B de marzo de 1965.

Nota 3: Para que se diera la vigencia inicial del Acuerdo, se exigió, por el mismo art. $9^{\circ}$, que, al menos, tres Estados lo firmaran sin reservas (sólo lo hizo Dinamarca) o hubieran depositado sus instrumentos de ratificación o aceptación. 
Nota 4: Liverpool, Manchester, Blackpool, el Cumberland y la isla de Man.

Nota 5: Edimburgo, Glasgow y el profundo estuario del Forth.

Nota 6: Como se sabe en Holanda el organismo emisor es la Nederlands Omroep Stichting (N.O.S.) y las organizaciones reciben un tiempo de emisión, excepto para los informativos que los realiza la NOS, en función de sus oyentes o telespectadores siendo la categoría A para un mínimo de 450.000 miembros, la B para 250.000 y la C para 100.000 como mínimo. El número se calcula en función del número de subscriptores. Tomado de EBU technical Review August 1977.

Nota 7: "Le Monde" 23 janvier 1979 p. 31; Correspondance de la presse 23 janvier 1979 .

Nota 8: Para ampliar la noticia consultar: "The Daily Telegraph" y "The Times", 21 march 1980 y el "Pueblo" y "Le matin" de la misma fecha y "El país", "ABC", "Le Monde", "Liberation" del 22 de marzo de 1980 y "Revue de l'UER Cahier Technique" $\mathrm{n}^{\circ} 180$ avril 1980.

Nota 9: Barco o vaporcito utilizado para la pesca en dos barcas apartadas la una de la otra que tiran de la red arrastrándola por el fondo, según "Le Monde" 24 août 1983 p. 12. 